\title{
OBSERVACIONES SOBRE EL PAPIAMENTO
}

Las siguiente notas, adición fonética al trabajo de Rodolfo Lenz ${ }^{1}$, fueron recogidas durante un viaje de La Guardia a Puerto Rico en un barco cuyos tripulantes eran en su mayor parte naturales de Curaçao. La lengua que hablaban entre ellos era normalmente el papiamento, pero podían servirse también con más o menos corrección del holandés, español e inglés. Fué examinada principalmente la pronunciación de un inteligente marinero de Wellemstad, Curaçao, quien después de ejercer durante mucho tiempo la profesión de zapatero en su ciudad y en otros lugares había entrado a servir como auxiliar de administración en los barcos de una compañía naviera de Nueva York. Compuso este sujeto un corto relato al cual pertenece el fragmento que adelante se transcribe.

Vogales acentuAdas.-La $a$ acentuada fué predominantemente de timbre medio: baca, clabo. Resultó palatal en araña, muraya, spada y manga. No fué velar sino media en calbo, pero pareció tan velarizada en trabáu 'trabajo', que prácticamente se podía oír trabóu. Lenz transcribió esto último. Se advirtió gran vacilación respecto al timbre de la $e$, la cual fué media en candela 'fuego' y frena 'brida', abierta en oreya 'oreja' y pretu 'negro', y cerrada en dede 'dedo' y lechi 'leche'. La o mostró en general timbre abierto: rosa, toro, boca, hobe 'joven', lomba 'espalda' y wowo 'ojo'. Predominó el tipo abierto o semiabierto en $i$, $u$ : clina 'crin', biki 'pico', pruga 'pulga', crus 'cruz'.

VOCALES INAGENTUADAS.-Se manifestaron corrientemente con sonido vacilante e impreciso. La $a$ de la sílaba inicial mostró tendencia palatal en cabái 'caballo' y camná 'caminar'; desarrolló marcadamente esta inclinación en maishi 'maíz' y masiar 'mucho', y' ofreció marcado sello posterior en trabóu. El timbre medio correspondió a esa misma vocal en patia 'sandía', cabritu, familia, també 'también'. Se oyó como $e$ en lembé 'lamer'. Fueron registrados con simple $s$ líquida, sin resto de vocal inicial, spada, stribu y stampia 'sello'. El desarrollo de una vocal silábica ocurrió en sunú, snú 'desnudo'. Se dió con fre-

1 Rodolfo Lenz, "El papiamento, la lengua criolla de Curaçao: La gramática más sencilla”, en $A U C h$, 2ạ serie, IV, 1926, págs. 695-768, 1023-109o; V, 1927, págs. $287-327,365-412,889-989$. 
cuencia el paso de $e$ a $i$ y de $o$ a $u$ tanto en la posición indicada como en fin de palabra: milón 'melón', buricu 'borrico', lechi 'leche', yabi 'llave'. Otros cambios notados fueron cu 'que' cun 'con', promer 'primer', sodá 'sudar', dede 'dedo' y tete 'ubre'. Fué corriente el ensordecimiento de la vocal final ante pausa en casos como cabritu, buricu, porco, maishi, bonchi 'habichuela'.

Diptongos.-Aparecieron en su forma normal en reina, seis, piedra, siegu 'ciego', muerte, suegu 'suegro'. La forma de 'veinte' fué binti. En el diptongo ue se observó intensa labialización de la $e$, la cual fué oída casi como ö en nuebe y webo. 'Bailar' apareció transformado en baliá y 'ciudad' en suidá. La semiconsonante se dió fundida con la palatal anterior en ñetu 'nieto' y masiar 'demasiado, mucho'. El acento fué situado sobre la $i$ en pia 'pierna, pie', y pasó desde el primero al segundo elemento en yu 'hijo' y cuchu' 'cuchillo'. 'Buey' se redujo a bue, con fuerte labialización de la $e$. 'Peor' y 'golpear' se dieron bajo la conocida forma de pió y golpiá. No ocurrió diptongación ni cambio de acento en maishi 'maíz' ni en baúl.

Consonantes $b, d, g$-Fueron regularmente oclusivas, como en español normal, en posición inicial absoluta y detrás de $m, n$. La $d$ se produjo como y africada en yenti 'diente'. En cualquier otra posición las consonantes indicadas se manifestaron con articulación vacilante. A Lenz le parecieron en general oclusivas. En el sujeto a que se refieren estas notas la forma oclusiva predominó en contacto con $r, l: c a$ britu, barba, barbuleta 'mariposa', yebra 'hierba', berde, tardi, birgui 'virgen'. Entre vocales fué más frecuente la forma fricativa: cabés 'cabeza', cabai 'caballo', spada, sodá 'sudar', ogá 'ahogar', yegá 'llegar', siegu 'ciego'. La oclusión reaparecía fácilmente al insistir sobre cualquiera de esos casos. La $v$ se identificó en toda ocasión con la $b$, unas veces como bilabial oclusiva (conversá, clabo, nuebi, yabi) y otras como bilabial fricativa (motivo, calbo, hobe 'joven'). No pareció que quedase ningún resto de $g$ en awa 'agua', yewa 'yegua', ni de la $b$ en wela 'abuelo, abuela'. La $d$ fué oída como $r$ en dos palabras muy repetidas: tur 'todo' y masiar 'demasiado'.

Fricativas $f, s, j$. - La $f$ presentó corrientemente articulación mixta entre labiodental y bilabial en familia, forti, frena y flor ${ }^{2}$. El sonido ordinario de la $s$ correspondió al tipo coronal postdental plano, con inclinación a la modalidad ápicoalveolar: asi, misa, sopi 'sopa'. En brasa 'brazo', sorsaca 'guanábana' y bisá 'avisar' pareció oírse una ápicoalveolar semejante a la castellana. La $s$ final de sílaba se reducía

$\therefore$ Respecto a la $h$ aspirada, fué perceptible, aunque débilmente, en hariña, hundi 'hundir', herru 'hierro', huma 'humo' y hartá 'hartar'; pareció reducirse a un simple ataque vocálico en ámber 'hambre' y asi 'hacer', y no se oyó de ningún modo en ogá 'ahogar', uzvi 'huir'. 
y atenuaba, sin llegar a desaparecer ni convertirse en aspiración. Se manifestó con sonido palatalizado en contacto con la vocal $i$ : nañish $i$ 'nariz', maishi 'maíz', masiar 'mucho', dushi 'dulce', lombrish 'cubo de la rueda (ombligo)', y en el tratamiento sion 'señor', con $o$ nasal. Fué normal el seseo: serca, gosá 'gozar', cabés 'cabeza'. Ejemplo análogo al de Stúñiga-Zúñiga apareció en stabachi 'azabache'. La $j$, pronunciada como leve aspiración en hobe 'joven', hende 'gente' ${ }^{3}$, resultó enteramente borrada en para 'pájaro', parabrua 'mochuelo (pájara bruja)', yu 'hijo', trabáu 'trabajo, wowo 'ojo', vieu 'viejo'.

Palatales.-En la africada $c h$ se observó mayor desarrollo del elemento fricativo que en el español ordinario, con la correspondiente reducción de la parte oclusiva: lechi, cuchara, chincha 'chinche'. La $y$ inicial sólo figuró como africada sonora, con terminación relativamente rehilante, en yenti 'diente' y yaca 'ardilla' 4 ; fué dicha con fricación regular sin rehilamiento en yewa 'yegua', yebra 'hierba', yabi 'llave', y con articulación más abierta y débil en boyo 'bollo', muraya y beyuda 'viuda'. La apócope de la palabra redujo la y a simple semivocal en cabái 'caballo', gai 'gallo', cabéi 'cabello' cucúi 'cocuyo'. Se suprimió la indicada consonante en posición intervocálica en contacto con la vocal $i$ : sía 'silla', rodia 'rodilla', semia 'semilla', brio 'brillo', puitu 'pollito'. No se hizo ninguna mención que revelara noticia de la $l l$.

Líquidas.-La $r$ simple fué pronunciada en cualquier posición con suave sonido fricativo. Resultó especialmente tenue ante otra consonante: muerte, barba, gordo, y fué eliminada en los infinitivos: mirá, mustrá, muri. Análoga inconsistencia pudo advertirse en la $l$, débil y reducida en calbo, golpiá, apenas perceptible en fin de palabra en baúl, clabel, y desaparecida en dushi 'dulce'. Dos casos de metátesis demostrativos de la preferencia por la sílaba libre fueron yebra 'hierba' y pruga 'pulga'. No se dió con abundancia el cambio entre $r y l$ representado por el último ejemplo. En general los dos sonidos se diferenciaron con regularidad, aparte de la $r$ interior de carné y la final de flor, intermedias entre $l$ y $r$, y la de calbón que fué una $l$ clara. Muy semejante a la de la $r$ simple fué la articulación de la $r r$, pronunciada como alveolar fricativa sin vibración de la punta de la lengua. Mostraba alguna mayor tensión y duración en principio de palabra, reina, rosa, rabo, y se oía en realidad como simple $r$ fricativa en buricu 'borrico', garoti 'garrote', kitara 'guitarra'.

NASALES.-La $n$ final de palabra se mostró de manera regular con

${ }^{8}$ La propagación de sonoridad que se observa en hende no ocurrió en yenti 'diente'. Lenz (art. cit:, V, 1927, pág. 385) señaló, entre otros casos, mondi 'monte', dera 'entera' y sanguro 'zancudo'.

${ }^{4}$ Otro caso de $y$ africada debido a la posición sintáctica ocurrió en con ye cabai 'con los caballos' en el texto transcrito. 
sonido velar débil, en gran parte absorbido por la vocal precedente: milón, calbón, fin. La $\tilde{n}$ no se produjo con pleno contacto dorsopalatal; su efecto era como el de una $y$ fricativa nasalizada cuya resonancia se extendía sobre las vocales inmediatas: araña, dañino, caña. En pronunciación espontánea, gallina, port. gallinha, fué pronunciada galia, con las dos últimas vocales nasalizadas y sin resto de $\tilde{n}$ ni de $y$; al hacer repetir la palabra reapareció la y nasal. La nasalización de las vocales se produjo en grado variable, así en ñetu, mustrá, maíshi, como en contentu, plantá, conversá. En comehén 'polilla', la influencia nasal invadió, además de las vocales, la aspiración de la $h$, pronunciada en unos casos como sorda y en otros como sonora. Acentuaba el efecto de la nasalización la frecuencia de la partícula de tratamiento, sion 'señor' con $s$ palatal y o nasalizada, y la forma pronominal africana nan 'ellos', con a nasalizada y la última $n$ velar.

IMPRESIÓN DE CONJUNTO.-En conjunto la pronunciación del papiamento produce impresión agradable por la suavidad de sus articulaciones, por el blando ritmo de su acento y por la melodía de su entonación. El compás de las palabras en los sujetos examinados era relativamente lento. El timbre de las vocales osciló entre límites considerablemente amplios. Las modificaciones de las consonantes se produjeron también entre márgenes de notoria extensión y en circunstancias variables. Tampoco la nasalización se manifestó con regularidad de proporción y medida. Todo esto daba lugar a que la imagen fonética de las palabras presentara alteraciones importantes no sólo de un individuo a otro sino en la boca de la misma persona. Es evidente que la fonética del papiamento se caracteriza por su inestabilidad. Es un habla de modulación grave, sin inflexiones rasgadas ni resonancias guturales. Un rasgo saliente es la nota oscura que resulta de la nasalización abundante y de la gran repetición de la u: bunitu, buricu, cunucu, tur 'todo', cun 'con', cu 'que', etc.

Transcripción.-El siguiente trozo del relato del marinero reúne gran parte de las particularidades señaladas en las notas precedentes:

Un dia mi a bai keiru na cunucu di sion Johans. Ora mi a jaga cerca sion Johans i su familia nan el a keda masiar contentoe. Pasobra ta hopi dia coe el no a mira mi, pa motivo coe mi señora moeri. Despues coe nos a conversa hopi nos a bai den cunucu, i sion Johans a camna rond mustrami toer lo que el a planta: masiar maishi, bonchi, pampuna, patia, melon, en fin, hopi fruta mas i nan toer tabata masiar berde, i su cabritoe, buricu, porko, carne, galiña, en fin toer su bestia nan ta gordo. El tin dos cabai masiar bunita. Ki ora coe sion Johans bine punda con ye cabai nan toer hende ta gusta $\mathrm{e}$ cabai pretoe manera stabachi ${ }^{5}$.

${ }^{5}$ Se ha conservado la escritura del relato, en letra grande y desigual, del mismo sujeto, con sus propias vacilaciones entre $o e$ y $u, b$ y $v, j$ y $y, c$ y $s, c$ y $k$. 


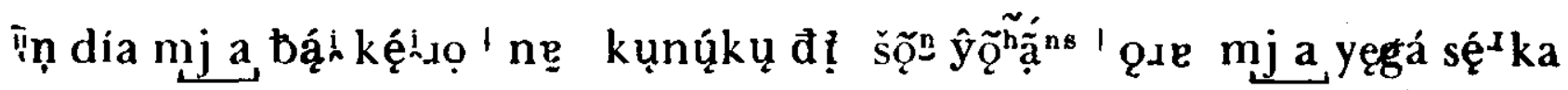

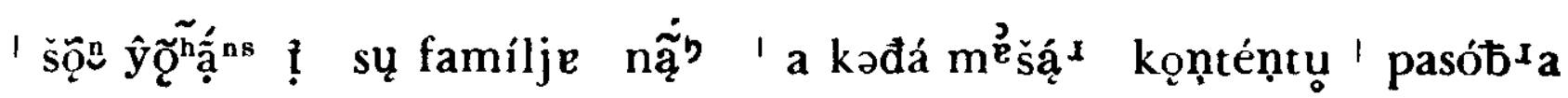
ta 'ọ́p̣̣ đía kwę nwa mị sá mẹ ' pa motíbọ kụ mị sẹnóxa a mụuị '

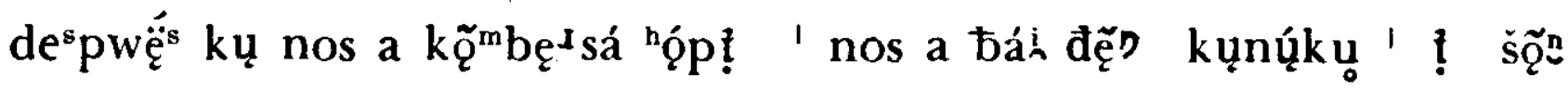

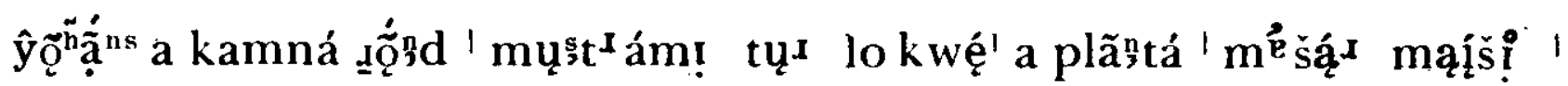

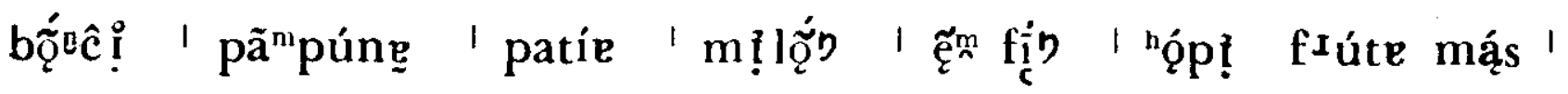

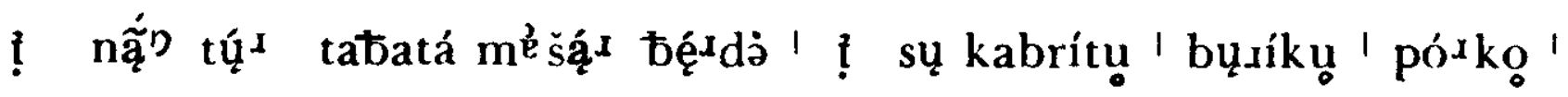

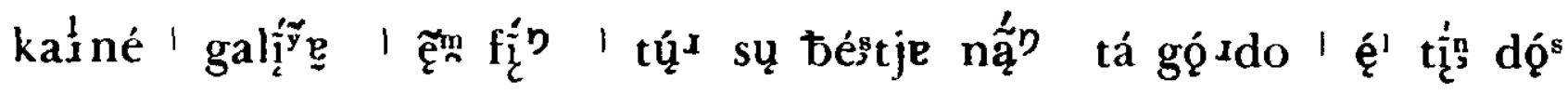

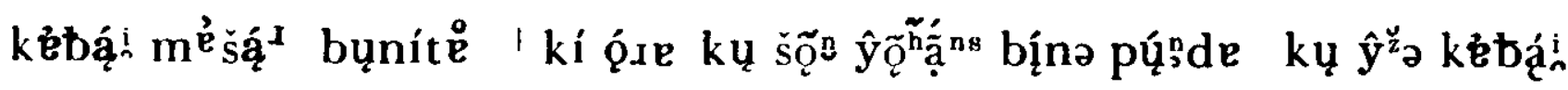

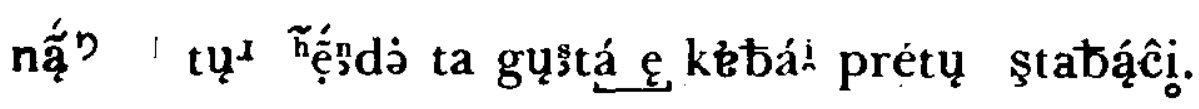

(Un día yo fuí a pasear al campo del señor Juan. Entonces llegué a casa del señor Juan y su familia. Él quedó muy contento, porque hacía muchos días que no me veía por motivo de la muerte de mi señora. Después que conversamos mucho fuimos al campo y el señor Juan dió una vuelta para mostrarme todo lo que él había plantado: mucho maíz, habichuelas, oyama, sandías, melones, en fin, muchas frutas más, y todas ellas estaban muy verdes, y sus cabras, borricos, puercos, carneros, gallinas, en fin, todos sus animales estaban gordos. Él tiene dos caballos muy hermosos. Cada vez que el señor Juan viene a la ciudad con los caballos, toda la gente admira el caballo, negro como azabache).

Expresiones sueltas: Bon nochi 'Buenas noches'. - Bon dia 'Buenos días'. - ¿Com bai? ‘Cómo le va?' - Masiar bon, danke 'Muy bien, gracias'. - ${ }_{6}$ Onda bo tabata ayera? '¿Dónde estuvo usted ayer?' - Ayera mi tabata na cunucu 'Ayer estuve en el campo'. - ¿Onda bo ta bai? 'AAdónde va usted?' - Mi ta bai punda 'Voy a la ciudad'. Ta wela ta bieu i su yu ta hobe 'Este abuelo es viejo y su hijo es joven'. - Si boyo tin manteca casca ta lombra 'Si el bollo tiene manteca la corteza brilla'. - Cuando uno cree que se le tiene en poco se suele decir: ¿¿Ki ku bo ta kere? ¿Mi ta casiu sin casipete? ¿QQué es lo que usted cree? ¿Que soy pajuil sin castaña?' - Nombres descriptivos: cabei di wowo 'cejas', renchi di orea 'pendientes', cuchú di sembra 'hoz', nañisi plat 'chato', nañisi pará 'narigudo', parasol di diablo 'hongo', da la coch 'saltamontes', cabés di maishi 'mazorca'.

Procedencia.-La isla de Curaçao, descubierta por el capitán Alonso de Ojeda en 1499, estuvo en poder de España hasta 1633 en que pasó a manos de los holandeses, los cuales la convirtieron en 
centro principal de comercio negrero ${ }^{6}$. Los pocos centenares de indígenas que la habitaban y los escasos españoles que componían su guarnición fueron sustituídos por los nuevos dueños holandeses y por la población de color. La lengua española desapareció de la isla con el cambio de dominio ${ }^{7}$. Los negros, procedentes de las colonias portuguesas del Oeste de África, hablaban portugués acriollado, con fuerte influencia africana. El intenso tráfico de negros durante los siglos XVII y xviII formó en Curaçao una densa población permanente, aparte de la que se detenía en la isla de manera transitoria. El papiamento se produjo en esos siglos, sin intervención del español, como dialecto afroportugués, aunque Curaçao nunca perteneciera a Portugal. El mismo dialecto se extendió a las islas próximas de Aruba y Bonaire. El sello portugués persiste en la nasalización, en la relajación de las vocales inacentuadas y en palabras como porta, porco, galiña, hariña, bon, també, antó 'então', ainda 'aún', etc.

INFLUENCIA DEL ESPAÑol.-Después de años de ausencia el español volvió a Curaçao, llevado en diferentes fechas, a partir de la segunda mitad del siglo xvir, por judíos sefardíes procedentes de las Guayanas, Madeira y Brasil ${ }^{8}$. La representación de tal lengua entre estos inmigrantes resultaría en cierto grado limitada por tratarse de forasteros que por su parte conocían también el portugués y acaso se servían con ventaja de esta lengua para alternar con la población de la isla. El español no debió empezar a ejercer efectiva influencia hasta que, a principios del siglo xix, núcleos importantes de venezolanos y colombianos trasladaron sus negocios y residencias a Curaçao por motivos políticos o económicos. Contribuiría al mismo efecto la facilidad con que los habitantes de la isla podían ejercitar el comercio en los lugares de las vecinas costas hispanoamericanas.

Aunque el idioma oficial es el holandés, las lenguas que más se hablan, tanto en Curaçao como en Aruba y Bonaire, son el papiamento entre la clase popular y el español en el comercio y en las relaciones sociales. Alejado de toda comunicación con el portugués, el papiamento ha acudido principalmente al español en las necesidades de su desarrollo lingüístico. Se calcula que en la actualidad

- M. Fernández de Navarrete, Colección de los viajes y descubrimientos que hicieron por mar los españoles desde fines del siglo xv, Madrid, 1829, vol. III, pág. 7 .

${ }^{7}$ Al tiempo en que los holandeses tomaron Curaçao, había en la isla 32 españoles y unos 1,400 indios. La mayor parte de los indios marcharon con los españoles a América del Sur. En 1635 sólo quedaban 75 indígenas y en 1695 casi habian desaparecido, según KARL SAPPER, “Ansiedlung von Europäern auf den Kleinen Antillen", en Schriften des Verein für Sozialpolitik, München, 1912 , CXLVII ${ }^{2}$, págs. 80-81.

${ }^{8}$ En 1654 se establecieron en Curaçao algunos judíos del Brasil, pero el contingente más numeroso, procedente de Madeira, no llegó hasta $1845^{-1} 84^{6}$ (cf. KARL SAPPER, art. cit., pág. 83). 
el $85 \%$ del léxico del papiamento es de origen español; al holandés se le atribuye el $5 \%$ y el resto se considera de procedencia portuguesa y africana. Una nueva influencia que ha ganado algún terreno en los últimos años es la del inglés.

Desacriollamiento.-La influencia del español no se ha limitado al vocabulario. La primitiva fonética afroportuguesa ha ido cediendo el campo a la española. Se han hecho predominantes las palabras con diptongación castellana: piedra, muerte; la $f$ inicial fué sustituída por la aspiración, la cual a su vez está siendo eliminada; la -it-portuguesa ha sido reemplazada por la $c h$ : ocho, nochi; están abriéndose camino las variantes fricativas de $b, d, g$; hay indicios de que se está reduciendo la nasalización de las vocales. Es de pensar que esta misma corriente podría observarse en la morfología si hubiera textos antiguos que permitieran comparar el papiamento moderno con el anterior al siglo XIX'.

Frente a la existencia de diversos dialectos criollos derivados del portugués y del francés, ha sido corriente referirse al papiamento como único dialecto de esta especie de origen español ${ }^{10}$. Como se ha visto, no se trata en realidad sino de un dialecto afroportugués desarrollado en Curaçao desde el siglo xvir bajo la dominación holandesa, al que el contacto con el español le ha hecho adquirir elementos que le han ido enriqueciendo, hispanizando y desacriollando. Hace años trataba Van Name de explicarse la causa de que entre los idiomas colonizadores modernos se distinga el español por no haber producido más dialecto criollo que el de Curaçao. La cuestión adquiere mayor interés ante el hecho de que ni el mismo papiamento pueda ser considerado como dialecto derivado de tal idioma.

Columbia University.

Tomás Navarro

- Se publican en papiamento periódicos, poesías, cuentos, gramáticas y diccionarios. En la bibliografía recogida por Lenz, las obras más antiguas son una cartilla escolar de 1843 y una traducción del Evangelio de San Matco, de 1844 .

${ }^{10}$ EI repetido supuesto de que el español aprendido por los negros había permanecido sin interrupción en Curaçao y de que el papiamento se había producido por el aislamiento en que tal población había vivido durante dos siglos respecto a los demás países hispánicos fué expuesto primeramente por A. VAN NAME, "Contributions to the Creole grammar," en Transactions of the American Philological Association, Philadelphia, I, 1869-70, pág. 125 . Lenz reconoció el origen portugués, pero, sin explicar cómo pudo efectuarse la colaboración, admitía que el español había participado también desde el principio en la formación del papiamento (véase su artículo citado, V, 1927, pág. 376). 